\title{
Primarily non-surgical management of osteomyelitis of the foot in diabetes
}

\author{
F. L. Game • W. J. Jeffcoate
}

Received: 20 December 2007 / Accepted: 2 February 2008 / Published online: 3 April 2008

(C) Springer-Verlag 2008

\begin{abstract}
Aims/hypothesis We examined the use of surgery and assessed the response to non-surgical management of osteomyelitis of the foot in diabetic patients.

Methods We reviewed the records of all patients presenting to a single specialist centre with osteomyelitis complicating a diabetic foot ulcer over a 5 year period. Details were extracted on antibiotic choice and treatment duration, hospital admission, incidence of minor and major amputation, and 12 month outcomes.

Results There were 147 patients, with mean age 64.7 years (66\% men). Of these, $26(18 \%)$ were admitted to hospital at the time of presentation and managed with intravenous antibiotics; the remainder were managed with oral antibiotics as outpatients. Surgery was undertaken because of life- or limb-threatening infection, or failure to respond, in 34 (23\%) patients (minor amputation 28, major amputation six patients). The remaining 113 were managed non-surgically. Remission was induced in $66(58.4 \%$ of 113$)$, while 35 (31\%) had a relapse. Of those experiencing relapse, 27 (77\%) achieved apparent arrest of the infection with a further course of antibiotics; six underwent minor and two underwent major amputation. Of all 113 whose infection was initially managed without surgery, apparent remission was achieved with antibiotics alone in 93 (82.3\%).

Conclusions/interpretation As these observations were made in an unselected case series, they give more insight into the respective roles of surgical and non-surgical management.
\end{abstract}

F. L. Game $(\bowtie) \cdot$ W. J. Jeffcoate

Foot Ulcer Trials Unit,

Department of Diabetes and Endocrinology,

Nottingham University Hospitals Trust,

City Hospital Campus,

Nottingham NG5 1PB, UK

e-mail: fgame@futu.co.uk
The results confirm that although urgent surgery is indicated in some patients, non-surgical management of those without limb-threatening infection is associated with a high rate of apparent remission.

Keywords Amputation - Antibiotics · Diabetes .

Diabetic foot · Gangrene · Infection · Osteomyelitis · Ulcer

\author{
Abbreviations \\ ERF established renal failure \\ MRSA methicillin-resistant Staphylococcus aureus \\ S(AD)SAD Size (Area, Depth), Sepsis, Arteriopathy, \\ Denervation
}

\section{Introduction}

Infection of the bone is a common complication of foot ulceration in patients with diabetes and presents a considerable therapeutic challenge [1]. Despite the existence of published guidelines $[2,3]$, approaches to the management of osteomyelitis of the foot in diabetic patients may vary quite widely, especially as few robust data are available to inform clinical practice [4]. Those working in different countries, healthcare systems and medical cultures may hold quite different views on the choice of antibiotics, their route and duration of administration, and on the place of surgery. Many specialists maintain that early surgical excision of all infected bone is essential, whereas others suggest that only necrotic bone should be removed. Others, again, believe that apart from possible limited debridement in the office or clinic, surgery may not be routinely necessary and should be reserved for patients who do not respond to treatment with antibiotics or in whom there are 
particular clinical indications. Henke and colleagues [5] argued strongly against such an approach when they reported the results of management of 224 cases $(80 \%$ with diabetes) of osteomyelitis affecting the digit, who had presented to a single centre over 7 years and who had at least 1 month of follow-up. Their view was summarised in the title of their paper: 'Osteomyelitis of the foot and toe in adults is a surgical disease: conservative management worsens lower extremity salvage'.

In support of a more conservative approach is the evidence from over 500 reported cases in whom the initial management was primarily non-surgical and in whom there was a mean rate of eradication of infection of over $60 \%$ [6]. A recent series reported from a single centre [7] reported apparent cure or remission in $80 \%$ of cases managed in this way. However, all of these data on non-surgical management derive from uncontrolled observational series and all may have been affected by case selection to the extent that patients in whom early surgery was undertaken because of disease severity at presentation may have been nonsystematically excluded. We therefore sought to examine the outcome of 147 consecutive cases in diabetic patients of osteomyelitis of the foot diagnosed at a single specialist centre in the UK between 1 January 2000 and 31 December 2004 , where the management policy was to use antibiotics alone whenever possible, although early surgical removal of infected tissue was undertaken if the severity of the infection was such that the all or part of the foot was judged non-viable.

\section{Methods}

The details of all patients and all lesions presenting to the specialist multidisciplinary outpatient diabetic foot service are recorded at the time of first attendance, with ulcers being classified according to the Size (Area, Depth) Sepsis, Arteriopathy, Denervation [S(AD)SAD] system as previously described [8]. The S(AD)SAD system allows definition of ulcer area, depth, limb ischaemia and neuropathy and the distinction of infection of soft tissue and of bone. All patients recorded as having osteomyelitis complicating a diabetic foot ulcer at first outpatient presentation between 1 January 2000 and 31 December 2004 were considered for inclusion in this analysis. If a patient had more than one episode, the first was selected. The details of each selected episode were checked by detailed case note review. However, as wound registration is only undertaken at the time of presentation of each new episode, patients who develop osteomyelitis during the course of management of a pre-existing ulcer are not readily identifiable from the clinic database. An electronic search was therefore made of the X-ray reports of all patients managed by the service during the same period. The case notes of those reported to have radiological changes compatible with a diagnosis of osteomyelitis were then examined by the authors, who are consultant specialists in the field, in order to establish whether a clinical diagnosis of osteomyelitis had also been made. The use of the specialist clinic register was approved by Nottingham University Hospitals Trust. It had previously been established by the Caldicott Guardian that approval of the Local Research Ethics Committee is not required for analysis of the outcome of routine management and for the publication of anonymised data derived from it.

The diagnosis of osteomyelitis was primarily clinical and was based on the presence of signs that were strongly suggestive: characteristic localised inflammation in association with a foot ulcer, with discharging pus and/or bone fragments, and evidence of disruption of bone cortex on plain X-ray. When the diagnosis was suspected on clinical grounds but not confirmed radiologically, symptoms were treated and X-rays were repeated at intervals until the diagnosis was established or excluded. Magnetic resonance imaging and occasional technetium and labelled white cell scans were undertaken in cases of continuing uncertainty, as well as in patients thought to have acute Charcot's disease rather than bone infection. Blood culture, haematological and biochemical tests were not undertaken on a routine basis. Bone biopsy was not performed.

Patients with more severe infection, who were systemically unwell or whose condition was complicated by significant co-morbidity, were admitted to hospital and had a broad-spectrum antibiotic regimen administered intravenously. Domiciliary intravenous treatment was not available. The philosophy of primary clinical management was to manage the infection non-surgically in the first instance, but operative debridement was performed if a collection of pus was suspected; minor amputation (surgery involving transverse excision of bone below the ankle) was performed if part or all of the forefoot was judged to be non-viable or if this was patient preference. Early major (transtibial or transfemoral) amputation was performed if it was judged that the limb was not salvageable. The remainder of patients were managed as outpatients, using an empirically chosen broad-spectrum antibiotic regimen. The usual oral antibiotic regimen chosen for patients who were not penicillin-allergic was amoxicillin/clavulanic acid (500/125 mg 8 hourly; 250/125 mg 8 hourly in those with established renal failure [ERF]). For patients who were allergic to penicillin, the usual first choice was clindamycin $300 \mathrm{mg} 6$ hourly plus either ofloxacin (400 mg twice daily) or ciprofloxacin $(500 \mathrm{mg}$ twice daily; dose halved in patients with ERF). The usual primary intravenous regimen was either a combination of gentamicin, amoxicillin/ ampicillin and metronidazole or clindamycin plus quinolone. Wounds were not routinely sampled for infecting 
bacteria unless pus or extruded bone was readily available; blood cultures were only undertaken in those who had systemic symptoms and signs. If a surface swab had been taken as part of standard nursing procedure in a patient in whom the diagnosis of osteomyelitis had been made on other criteria, and yielded methicillin-resistant Staphylococcus aureus (MRSA), suitable agents were added to the regimen or substituted for other antibiotics. Such agents included trimethoprim $200 \mathrm{mg}$ twice daily (100 mg twice daily in ERF patients), doxycycline (100 mg twice daily), fusidic acid (500 mg three times per day) and rifampicin (300 mg 6 hourly). If the clinical response was judged to be incomplete, microbiological sampling of pus, deep tissue by curettage or aspirate, or of extruded bone was undertaken and the antibiotic regimen was adjusted on the basis of the results. Associated ulcers were managed by regular office debridement, cleansing, dressing with non-adherent dressings and off-loading with non-removable casts wherever possible, according to international guidelines [9].

Hospitalised patients were transferred to oral therapy once the wound showed signs of clinical improvement, allowing early discharge home. Outpatient therapy with oral antibiotics was continued for at least 6 weeks. Outpatients were reviewed in the specialist multidisciplinary clinic at intervals of 1 to 4 weeks. When the infection was judged on clinical grounds to have either been eradicated or gone into clinical remission, antibiotic therapy was discontinued, with the patient remaining under close surveillance. If signs of local inflammation recurred, antibiotic therapy was restarted and continued for a further period of weeks or months.

The primary endpoint was patient survival with limb intact (undergoing neither minor nor major surgery) at 12 months after the point at which the clinician felt the infection had been eradicated (either the discontinuation of antibiotics or after the initial surgical intervention). Other endpoints included the incidence of ipsilateral minor and major amputation and death.

\section{Results}

The diagnosis of osteomyelitis was made in 113 patients at the time of registration. In 34 other patients diagnosis of osteomyelitis was made during follow-up after initial attendance of the specialist foot clinic. Mean age of these 147 patients, 97 (66\%) of whom were men, was 64.7 (range $30-91)$ years. Thirty $(20 \%)$ of these patients had type 1 diabetes and $114(78 \%)$ had type 2 disease; in three cases diabetes type was not recorded. In $95(65 \%)$ cases, the infection was confined to the toes.

All 147 patients received antibiotics as part of their initial management. In 121 (82\%) patients, the antibiotics were administered orally, while $26(18 \%)$ were admitted to hospital and received antibiotics intravenously. In 34 patients the infection either failed to respond to initial therapy or was judged severe enough to threaten either life or limb; these patients underwent amputation (minor 28; major 6). The remaining 113 (77\% of 147) had no surgery as part of initial management (Fig. 1).

The initial choice of oral therapy comprised coamoxiclav (amoxicillin/clavulanic acid) in 57 (35.4\%), while $52(38.8 \%)$ had clindamycin in combination with a quinolone (ofloxacin or ciprofloxacin). Of those given oral treatment, $47(32 \%)$ changed oral antibiotics at a later stage, either on the basis of the results of microbiological sampling or because of limited clinical response. Secondline oral treatment regimens involved changing to clindamycin plus quinolone in 15 or to co-amoxiclav in 12 patients. Fusidic acid, doxycycline and trimethoprim were added to initial therapy in five, three and one cases, respectively. Seven patients received third-line therapy with regimens including fusidic acid, doxycycline and rifampicin. The median length of initial oral treatment for all patients was 61 days (range 3-349 days). Median length of intravenous antibiotic treatment was 16 days (range 144 days).

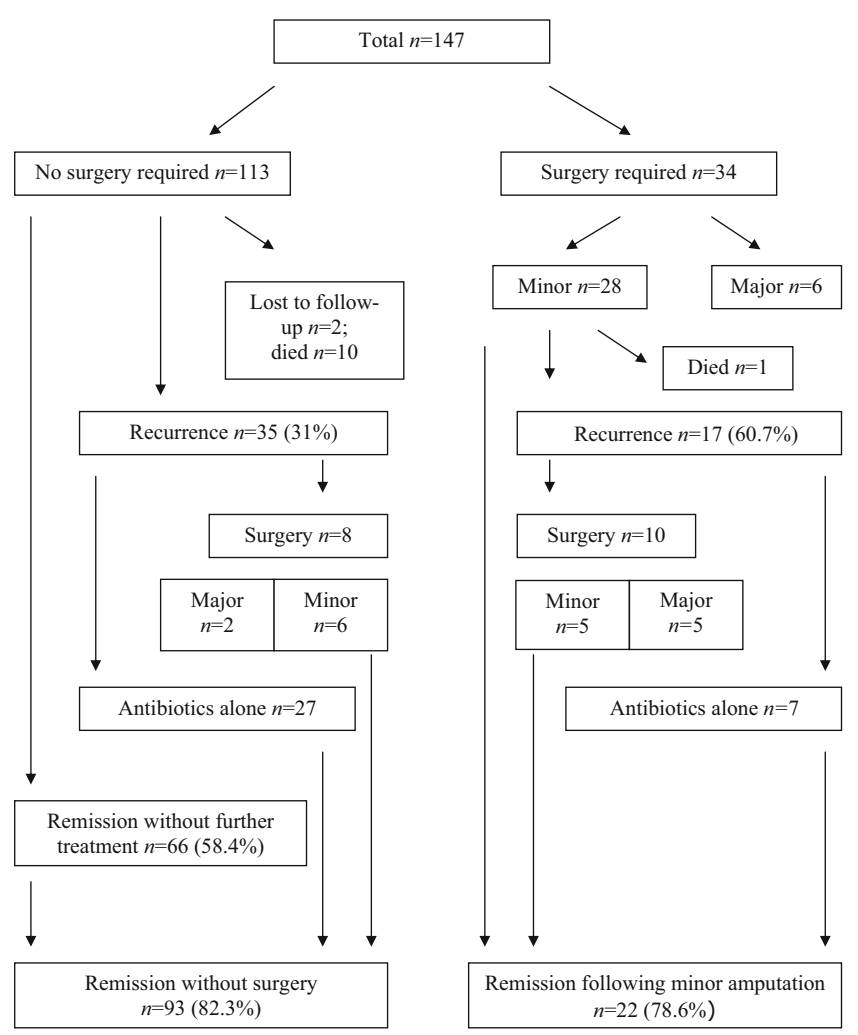

Fig. 1 Flow diagram illustrating outcome in 113 cases of osteomyelitis managed initially without surgery and in 34 patients who required surgery as part of the initial management. Minor, minor amputation (below the ankle); major, transfemoral or transtibial amputation 
Of patients treated with antibiotics alone, lasting remission at 12 months was observed in 66 (58\%), while recurrence or reactivation following initial discontinuation of therapy was seen in 35 (31\%) patients (Table 1). Remission was established in 27 of these $35(77 \%)$ following a second course of oral treatment, while six underwent minor amputation and two had major amputation. Of the 28 managed initially with antibiotics plus minor surgery, there was a recurrence in 17 (61\%), in five and seven of whom remission was achieved after further minor surgery and antibiotics alone respectively, while five underwent major amputation. Overall, 93 of $113(82 \%)$ patients who were managed non-surgically were in remission at 12 months, while in patients managed with minor amputation, remission was achieved in 22 of 28 (78.6\%). Major amputation was undertaken in 13 (9\%) patients. Eleven patients died, ten $(8.8 \%)$ in the non-surgical and one $(3.0 \%)$ in the surgical group $\left(p=0.27, \chi^{2}\right)$. Two in the nonsurgical group were lost to follow-up.

No association was found between baseline S(AD)SAD score for arteriopathy and either initial management $\left(\chi^{2}\right.$ test, $p=0.35$ ) or those with final operative intervention (five major amputations in patients with intact pulses, one major amputation in a patient whose pulses were reduced and six in patients whose pulses were absent, $\chi^{2}$ test, $p=0.37$ ). However, a significant association was found between $\mathrm{S}(\mathrm{AD}) \mathrm{SAD}$ arteriopathy score and death in the follow-up period $(p=0.039)$.

\section{Discussion}

The data presented here are derived from a consecutive series, which was managed in a single specialist centre and is unlikely to be significantly affected by selection bias. The mean age of the population and the ratio of men to women was that which would be expected. The philosophy of management in this centre was to attempt to eliminate active infection with prolonged courses of oral antibiotics whenever possible. Patients with more severe infection

Table 1 Twelve month outcomes in patients managed with antibiotics alone, compared with those managed with antibiotics plus minor amputation at initial presentation

\begin{tabular}{lcl}
\hline Outcome & $\begin{array}{l}\text { Antibiotics } \\
\text { alone }(n=113)\end{array}$ & $\begin{array}{l}\text { Antibiotics with minor } \\
\text { amputation }(n=28)\end{array}$ \\
\hline $\begin{array}{l}\text { No recurrence of active } \\
\text { disease }\end{array}$ & $66(58.4)$ & $10(35.7)$ \\
$\begin{array}{l}\text { Recurrence or reactivation } \\
\text { Died }\end{array}$ & $35(31.0)$ & $17(60.7)$ \\
Lost to follow-up & $10(8.8)$ & $1(3.6)$ \\
\hline
\end{tabular}

Values are $n(\%)$ were hospitalised for intravenous therapy, combined when judged necessary with either minor or major amputation. This policy was associated with apparent eradication of active disease without any form of amputation in 93 patients, representing $63 \%$ of the total series and $82 \%$ of the 113 patients whose initial management did not involve surgery. Of the 113 patients initially managed without surgery, a relapse was observed in 35 (31\%); however, this relapse responded to further non-surgical management in 27 , with only eight undergoing second-phase surgery. The high rate of recurrence (18 of 28, 64\%) following early minor amputation is probably explained by these patients having had more severe disease at presentation. However, remission was induced in the majority of these, either by further minor amputation or by the use of antibiotics.

Interestingly, there were more deaths (not statistically significant) in the group of patients who had conservative management initially. At first sight it may appear that risk of death is potentially higher when conservative management is chosen. On the other hand, many elderly patients are frail (especially those with significant and inoperable peripheral arterial disease) and surgery, for them, may be neither safe nor in their best interests. We have not excluded such patients from this series, which represents a consecutive series of patients who presented to our service. Given the observed association between grade of ischaemia and risk of death, we feel that this is the likely explanation for this finding.

These results compare favourably with the only other recent report on an unselected series of similar size $(80 \%$ of whom had diabetes) [5]. Although the patients were younger in that earlier study (mean 57 years, compared with 65 years in the present series), the eventual incidence of major amputation was much higher at 25\% [5]. Our experience therefore differs from that of the authors of that work, who concluded that 'conservative management worsens lower extremity salvage'. It is possible that the population managed in the earlier series had more severe disease at the time of presentation, but whatever their cause, these differences highlight the difficulties of extrapolating results from one centre to another. Superficially, however, the data from the current study suggest that primarily nonsurgical (or conservative) management can be successful in the majority of cases.

These conclusions echo those of many other groups who have shown that non-surgical management of bone infection of the foot in diabetic patients can be eliminated or arrested by non-surgical management in the majority of cases [7, 10-18]. However, the earlier reports were more in the nature of proof-of-concept studies. Moreover, because the current series was consecutive, it is possible to identify in how many patients such a policy is simply not practicable because of the severity of the disease at presentation. 
The inclusion of these additional cases strengthens the case for suggesting that treatment with antibiotics alone can reasonably be attempted in the majority of cases, even though the choice of management of each case must always be determined by the individual circumstances and there are clearly some patients in whom surgery is urgently indicated. It is of interest that the apparent cure rate in patients who did not have surgery as part of their initial management $(82.3 \%)$ was very similar to that reported in another large series reported recently by Embil et al. [7].

The prolonged use of broad-spectrum antibiotics is associated with an increased risk of infection with Clostridium difficile or the emergence of resistant strains of bacteria, such as MRSA. We did not specifically look for this in this population, although none of the patients in this cohort developed infection with $C$. difficile as far as we are aware We have previously reported an increasing prevalence of colonisation of foot ulcers with MRSA between the year 2002 (10\% patients) and 2004 (16.7\% patients). While it is not possible to exclude the possibility that this relates in part to antibiotic prescribing, we found that by far the strongest association with MRSA colonisation was previous admission of the patient to hospital. The risk of long-term antibiotics in an outpatient setting therefore has to be weighed against the risks of admission for surgery in the UK. Reassuringly, data from these same series showed no association between the isolation of MRSA and poor clinical outcome (healing of ulcers, amputation or death) [19].

It should be noted, however, that observational studies of osteomyelitis such as this are weakened by difficulties of case definition, especially as there are currently no agreed criteria by which bone infection, when clinically suspected, can be either diagnosed or excluded with confidence [4]. While it is acknowledged that reliance simply on clinical grounds with confirmatory imaging may be inadequate for rigorous scientific studies, these are the criteria used as the basis for diagnosis in routine clinical practice around the world, a fact that enhances the external validity of our observations. Bone biopsy (for microbiology and histology) is frequently referred to as the gold standard for diagnosis, but in practice it is only undertaken infrequently. Moreover, it is acknowledged that: (1) sampling errors may produce misleadingly negative results of microbiological culture; and (2) there has been no comparison of the histological manifestations of osteomyelitis and the condition with which it is most likely to be confused, acute Charcot's disease of the foot. It may, indeed, be impossible to distinguish osteomyelitis from acute Charcot's disease in clinical practice, since both occur in patients with neuropathy and relatively intact peripheral circulation, and both are associated with local inflammation and cortical bone disruption. It is therefore possible that a number of cases of diagnosed osteomyelitis may in fact have been instances of acute Charcot's disease; this is particularly likely when the changes are localised to the forefoot. Even though acute Charcot's disease is much rarer than osteomyelitis, such mistaken diagnoses may obviously have contributed to the apparently high response to non-surgical management. On the other hand, early amputation would not be good management of acute Charcot's disease and it could be argued that the unnecessary prescription of antibiotics for non-existent infection was the preferable option.

These and other issues, including the adverse effects of prolonged administration of antibiotics, can only be addressed in a randomised, controlled trial. Such a trial must consider the difficulties encountered in case definition, antibiotic choice and the problem of determining when infection has been effectively eradicated or arrested. Patient-centred outcomes (such as function, mood and satisfaction) and economic issues should also be assessed. Such a trial, however, can only be undertaken in a country or culture where early surgery is currently regarded as the intervention of choice, but also a country where clinicians are prepared to accept that a case can be made for deferring surgery until it is shown that nonsurgical treatment has failed. Randomisation to early surgery would be difficult to justify in a centre such as our own, where the prevailing belief was that early surgery was not necessary in the majority of patients. There will, however, be no certainty on the relative role of surgery and of antibiotics in the management of osteomyelitis of the foot in diabetic patients, until such a controlled prospective study has been conducted.

Acknowledgements We thank P. Ince and S. Kadhim for help in extraction of details from the clinic database. We also thank B. Kirk and V. Savage for their continuing support and for enabling us to complete this analysis.

Duality of interest The authors declare that there is no duality of interest associated with this manuscript.

\section{References}

1. Lavery LA, Armstrong DG, Wunderlich RP, Mohler MJ, Wendel CS, Lipsky BA (2006) Risk factors for foot infections in individuals with diabetes. Diabetes Care 29:1288-1293

2. Lipsky BA (2004) A report from the international consensus on diagnosing and treating the infected diabetic foot. Diabetes Metab Res Rev 20(Suppl 1): S68-S77

3. Lipsky BA, Berendt AR, Deery HG 2nd et al (2004) IDSA Guidelines: diagnosis and treatment of diabetic foot infections. Clin Inf Dis 39:885-910

4. Berendt RA, Peters EJG, Bakker K et al (2008) Diabetic foot osteomyelitis: a progress report on diagnosis and a systematic review of treatment. Diabetes Metab Res Rev. DOI 10.1002/ dmrr. 836

5. Henke PK, Blackburn SA, Wain RW et al (2005) Osteomyelitis of the foot and toe in adults is a surgical disease: conservative management worsens lower extremity salvage. Ann Surg 241:885-894 
6. Jeffcoate WJ, Lipsky BA (2004) Controversies in diagnosing and managing osteomyelitis of the foot in diabetes. Clin Infect Dis 39 (Suppl 2):S115-S122

7. Embil JM, Rose G, Trepman E et al (2006) Oral antimicrobial therapy for diabetic foot osteomyelitis. Foot Ankle Int 27:771-779

8. Treece KA, Macfarlane RM, Pound N, Game FL, Jeffcoate WJ (2004) Validation of a system of foot ulcer classification in diabetes mellitus. Diabet Med 21:987-991

9. International Working Group on the Diabetic Foot/Consultative Section of the IDF (2007) Practical guidelines on the management and the prevention of the diabetic foot. http://shop.idf.org/catalog/ product_info.php?products_id=68. Accessed 21 February 2008

10. Bamberger DM, Daus GP, Gerding DN (1987) Osteomyelitis in the feet of diabetic patients. Long-term results, prognostic factors, and the role of antimicrobial and surgical therapy. Am J Med 83:653-660

11. Nix DE, Cumbo TJ, Kuritsky P, Devito JM, Schentag JJ (1987) Oral ciprofloxacin in the treatment of serious soft tissue infection: efficacy, safety and pharmacokinetics. Am J Med 82(Suppl):146215

12. Peterson LR, Lissack LM, Canter K, Fasching CE, Clabots C, Gerding DN (1989) Therapy of lower extremity infections with ciprofloxacin in patients with diabetes mellitus. Am J Med $86: 801-807$
13. Ha Van G, Siney H, Danan JP, Sachon C, Grimaldi A (1996) Treatment of osteomyelitis in the diabetic foot. Contribution of conservative surgery. Diabetes Care 19:1257-1260

14. Venkatesan P, Lawn S, Macfarlane RM, Fletcher EM, Finch RG, Jeffcoate WJ (1997) Conservative management of osteomyelitis in the feet of diabetic patients. Diabet Med 14:487-490

15. Pittet D, Wyssa B, Herter-Clavel C, Kursteiner K, Vaucher J, Lew PD (1999) Outcome of diabetic foot infections treated conservatively: a retrospective cohort study with long-term follow-up. Arch Intern Med 159:851-856

16. Eneroth M, Larsson J, Apelqvist J (1999) Deep foot infections in patients with diabetes and foot ulcer: an entity with different characteristics, treatments, and prognosis. J Diabetes Complications 13:254-263

17. Senneville E, Yazdanpanah Y (2001) Cazaubiel M et al Rifampicin-ofloxacin oral regimen for the treatment of mild to moderate diabetic foot osteomyelitis. J Antimicrob Chemother 48:927-930

18. Yadlapalli N, Vaishnar A, Sheehan P (2002) Conservative management of diabetic foot ulcers complicated by osteomyelitis. Wounds 14:31-35

19. Game FL, Jeffcoate WJ (2006) MRSA isolation from diabetic foot ulcers-does it affect healing and is the problem getting worse? (Abstract) Diabetes 55(Suppl 1):99-OR 